Article

\title{
Optimization of Energy Management Strategy and Sizing in Hybrid Storage System for Tram
}

\author{
Yu Wang, Zhongping Yang * and Feng Li \\ School of Electrical Engineering, Beijing Jiaotong University, Beijing 100089, China; \\ ywangelec@bjtu.edu.cn (Y.W.); 17126003@bjtu.edu.cn (F.L.) \\ * Correspondence: zhpyang@bjtu.edu.cn; Tel.: +86-159-0140-6076
}

Received: 5 March 2018; Accepted: 22 March 2018; Published: 26 March 2018

\begin{abstract}
In order to design a well-performing hybrid storage system for trams, optimization of energy management strategy (EMS) and sizing is crucial. This paper proposes an improved EMS with energy interaction between the battery and supercapacitor and makes collaborative optimization on both sizing and EMS parameters to obtain the best working performance of the hybrid storage system. This paper demonstrates the whole process of the improvement of the EMS, the traction calculation, the parameter optimization, and the sizing optimization based on real tram and line conditions. The improved strategy is proven to be superior as it guarantees normal operation and avoids shutdown conditions when the supercapacitor is at a low voltage. Furthermore, the optimized sizing result largely decreases the weight of the storage system and obtains a long battery lifespan.
\end{abstract}

Keywords: hybrid storage system; sizing; energy management strategy

\section{Introduction}

As a kind of irreplaceable transportation in cities, the tram has experienced a process of development, decline, and innovation. From 1985, trams have been revived and have undergone fast development, thus becoming a significant symbol of modern cities. Modern trams have a higher speed, larger passenger volume, and higher comfort level than old trams and have become an important part of urban public transportation alongside buses and the subway. There are many new technologies in the development of modern trams, such as low floor technology, bogie technology, and non-grid power supply technology [1-3]. There are two kinds of non-grid power supply technologies: sectional ground power supply technology and on-board energy storage technology. The more commonly used is on-board energy storage technology. There are some similarities between a tram with on-board energy storage and an electric vehicle. However, there are also some differences. Trams have a large carrying capacity, high power and energy demand, and relatively fixed operating conditions. The common on-board energy storage system of trams includes a battery system, a supercapacitor system, a flywheel system, a hybrid system of an internal combustion engine and battery or supercapacitor, a battery, and a supercapacitor hybrid power system. Batteries and supercapacitors are the most widely used vehicle power systems, and they first appeared on trams in Europe [4] and Japan. In recent years, China's trams have also had a lot of applications: the Guangzhou Haizhu line uses a supercapacitor as the only power source; the Nanjing Hexi line and Jiangsu Huaian line only use batteries. However, battery-only storage system cannot have both high velocity and long lifespan because the battery's power density is lower than the supercapacitor, the high-power configuration will cause heavy weight, and it cannot discharge at high rate both for considerations of security and lifespan. In contrast, a supercapacitor-only storage system has high velocity and long lifespan, but the distances between the charging stations need to be very short given that if the supercapacitor's energy density is too low and the configuration cannot be very large, so it lacks endurance. To further improve the energy 
density and power density as well as the endurance of the energy storage system and reduce the volume and weight, more researchers have worked on hybrid energy storage systems with a battery and a supercapacitor.

EMS is an allocation standard of different energy storage systems and decides the performance of the hybrid energy storage system. There are many types of strategies used in engineering, such as the logic threshold approach $[5,6]$, which switches working states based on voltage thresholds, power thresholds, or something else. The strategy is simple and easy to control. However, the method generally relies on engineering experience and a lack of flexibility. Another popular method is the frequency decoupling strategy, which splits power based on defined frequency where the supercapacitor works with high frequency power requirements and the battery works with the low part [7]. This strategy is commonly used in electric vehicles where the supercapacitor is used as an auxiliary power supply system to provide and absorb high-frequency power. The main aim of the strategy is to lighten the pressure of the battery and extend the lifetime. Furthermore, there is another heuristic EMS based on fuzzy logic rules to classify working states $[8,9]$. The fuzzy logic rules have been used for a long time, so it has an accomplished theory and robustness. The above-mentioned methods can be applied in trams. However, all of the methods concentrate on neither the output characteristics of the hybrid storage system nor the lifespan of the battery, and none of them have made full use of the complementation between battery and supercapacitor. When the tram is in an emergency such as traffic jam or something else that makes the supercapacitor run out of energy, the tram can only slow down or stop.

Sizing is the key step of the tram's hybrid storage system optimization, and it has an impact on the characteristics of the energy storage system. Sizing seems to only influence the weight and volume of the hybrid storage system, nevertheless, the initial cost, life-cycle cost, battery lifespan, vehicle efficiency, and other elements are relative to sizing optimization. The procedure can be simple if it only considered the boundary conditions such as energy consumption, peak power, weight limitation, and terminal voltage [10-12]. Most of the sizing is based on certain EMS. The configuration result satisfies all the demands of operation and design; however, it is not the optimized result. Considering further optimization within the limitations, some papers have compared and selected the optimized sizing results by traverse method or algorithms $[13,14]$. However, the sizing optimization can influence the effect of the EMS as the parameters in the strategy are not always the best applied in a system with different sizing.

Most studies have used the defined EMS in the sizing optimization but have ignored the interaction between them. Only the collaborative optimization can make the energy storage system obtain a better working state. Based on the above matters, the contributions of this paper are as follows: The collaborative optimization is divided into three steps in the paper, as is shown in Figure 1. The first step proposes the improved EMS with energy interaction. The second step is to make optimization and obtain the optimal parameters in EMS. The third step is to make a multi-objective optimization of sizing and obtain a hybrid storage system with the best effect under this EMS. These three steps result in a system with relatively light weight, long lifespan, and good characteristics. Additionally, the EMS with energy interaction guarantees normal operation all the time, even in emergencies.

This paper is organized as follows. Section 2 provides the model of the hybrid storage system. Section 3 introduces the traction calculation procedure for the tram. Section 4 puts forward the improved EMS with energy interaction. Section 5 introduces the detailed process of collaborative sizing optimization. A detailed optimization procedure for the sizing and parameters in EMS based on real condition of trams and lines as well as simulation analysis are shown in Section 6. Section 7 presents the conclusions of the paper. 


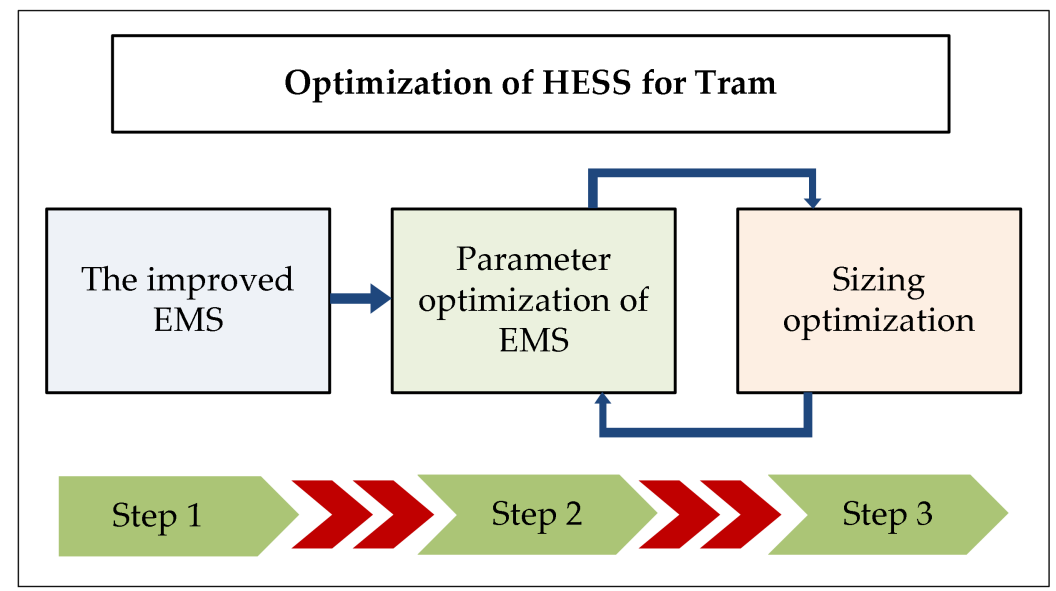

Figure 1. Optimization steps for hybrid storage system.

\section{Hybrid Storage System of Tram}

A tram's hybrid power system mainly consists of an energy storage system and a motor system. The motor system is connected to the DC bus through the inverter, whose power is all from the hybrid energy storage system. The storage system charges and discharges by receiving the control signal according to the requirement of the motor so that it can provide traction and auxiliary power and absorb regenerative braking power [15]. DC/DC converter is one of the key parts of a hybrid power system and it is one of the causes of system loss. To improve the efficiency of DC/DC and reduce the switching loss and impedance loss as well as the ripples, researchers have put forward many new ideas including increasing the bridge arm, improving the topology [16], using isolated switching devices, and so on. Common topologies for hybrid systems are shown in Figure 2 [17], which is mainly divided into single DC/DC topology (in Figure 2a,b) and double DC/DC topology (in Figure 2c,d).

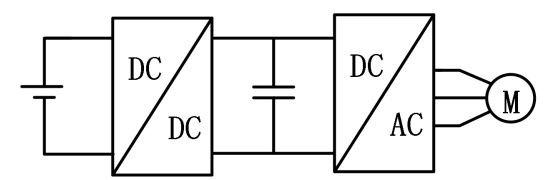

(a)

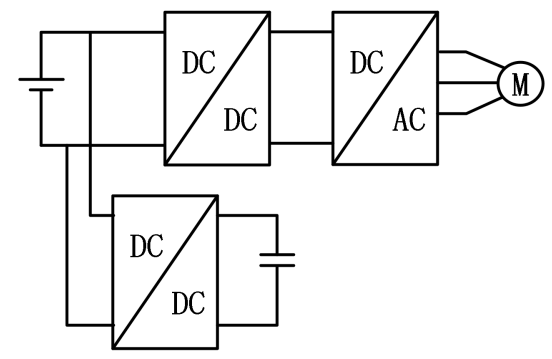

(c)

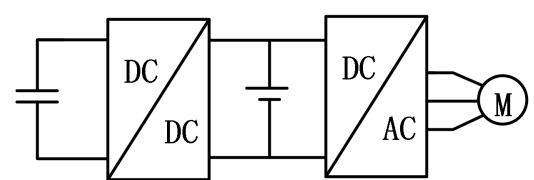

(b)

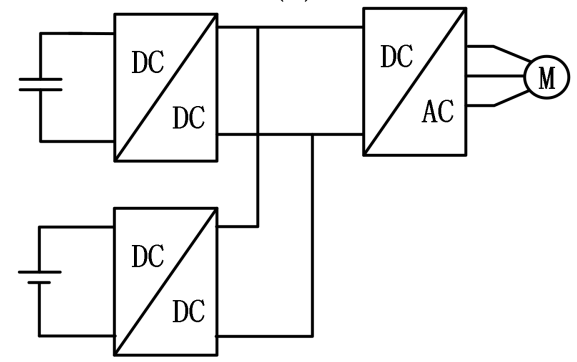

(d)

Figure 2. Common topologies for a hybrid power system. (a) Single DC/DC topology with controllable supercapacitor. (b) Single DC/DC topology with controllable battery. (c) Two-stage DC/DC topology. (d) Double DC/DC topology.

The single DC/DC topology can control the power distribution of an energy storage device, and the hardware control is simple and the programming is convenient. In the topology shown in Figure $1 \mathrm{a}$, the supercapacitor is directly connected to the DC bus, which reduces the power level of the DC/DC and helps to reduce the volume and weight of the energy storage system. However, due to the volatile voltage fluctuation of the supercapacitor, the storage's stability and efficiency are weakened. 
As shown in Figure 2b, the battery in the topology is directly parallel to the DC bus, and the bus voltage no longer fluctuates. However, when compared with the topology in Figure 1a, the power level of the DC/DC increases. Thus, the topology makes an increase in volume and weight.

The double DC/DC topology can control two kinds of energy storage devices at the same time, whose algorithm is more complex, but the control precision is high, the bus voltage fluctuation is small, and the system efficiency is high. At the same time, as one of the storage packs is directly connected to the DC bus in the single topology, the sizing optimization of the system is limited. Nevertheless, the topology with double DC/DC can reduce the volume and weight of the energy storage system through the further sizing optimization. Compared with Figure 2c, Figure $2 d$ is more favorable for control and has a wider range of application. The tram used in this paper used the topology shown in Figure $2 \mathrm{~d}$, and the structure of the tram and its on-board hybrid power system is shown in Figure 3.

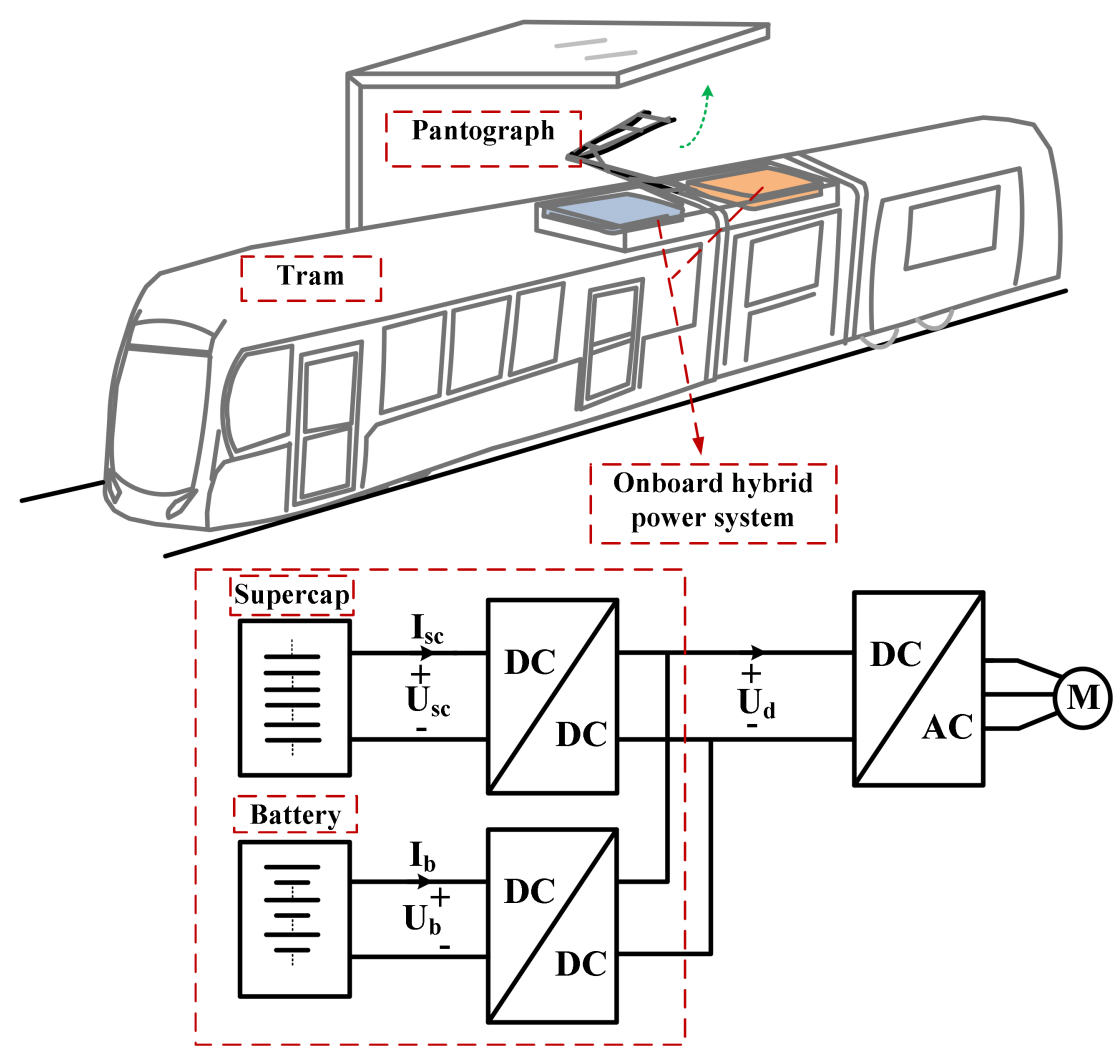

Figure 3. Topology of the hybrid energy storage system.

The relationship between the output power and the demand power of the hybrid storage system can be expressed as follows by the simplified nonlinear formula:

$$
P_{r e q}=\left\{\begin{array}{l}
\left(I_{b} \cdot U_{b}+I_{s c} \cdot U_{s c}\right) \cdot \eta_{d c}, P_{r e q} \geq 0 \\
\left(I_{b} \cdot U_{b}+I_{s c} \cdot U_{s c}\right) / \eta_{d c}, P_{r e q}<0 \\
k=1,2, \ldots, N
\end{array}\right.
$$

where $P_{r e q}$ is the required power from the DC bus. $P_{b}$ and $P_{s c}$ are the power requirements of the battery and supercapacitor, respectively. $\eta_{d c}$ is the efficiency of every DC/DC converter. $I_{b}$ and $I_{s c}$ are the currents of battery and supercapacitor, respectively. The battery model is based on the "Thevenin" 
battery model [18], as is shown in Figure 4a. The relations among current and voltages are shown in the following equations.

$$
\left\{\begin{array}{l}
U_{b}=U_{b o}-U_{b p}-I_{b} \cdot R_{b 0} \\
I_{b}=\frac{U_{b p}}{R_{b p}}+C_{b} \cdot \frac{d U_{b p}}{d t}
\end{array}\right.
$$

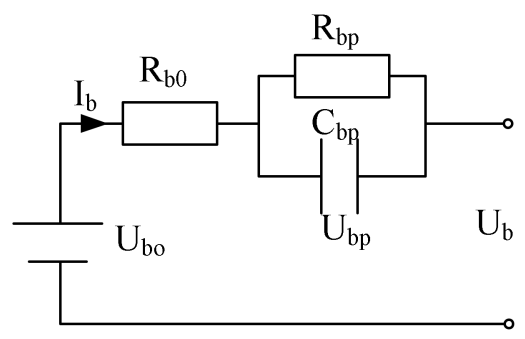

(a)

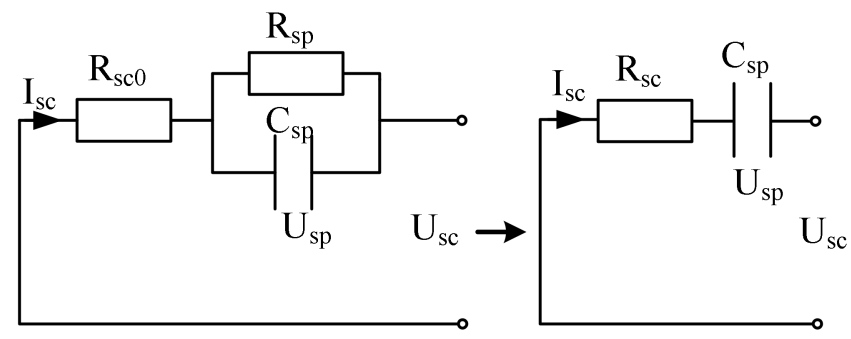

(b)

Figure 4. Models of battery and supercapacitor. (a) Battery model. (b) Supercapacitor model.

And the model of supercapacitor could be simplified as a "RC" capacitor model. The relations are shown in Equation (3):

$$
\left\{\begin{array}{l}
U_{s c}=-U_{s p}-I_{s c} \cdot R_{s c} \\
I_{s c}=C_{s p} \cdot \frac{d U_{s p}}{d t}
\end{array}\right.
$$

The equations of states of charge (SOC) are shown below:

$$
\left\{\begin{array}{l}
S O C_{b}=S O C_{b 0}-\frac{\int_{0}^{t} I_{b} d t}{Q_{b 0}} \\
S O C_{s c}=\frac{E_{s c}}{E_{s c \max }}=\frac{0.5 \cdot C_{s p} \cdot U_{s c}^{2}}{0.5 \cdot C_{s p} \cdot U_{s c \max }^{2}}=\frac{U_{s c}^{2}}{U_{s c \max }^{2}}
\end{array}\right.
$$

where $S O C_{b 0}$ is the initial $S O C$ of battery, and $Q_{b 0}$ is the battery cell's charge at the $S O C$ of $100 \%$. Additionally, to ensure the quality of the storage system, the voltages and SOCs are limited to a certain range, and the formula is expressed as follows:

$$
\left\{\begin{array}{l}
I_{b \min }<I_{b}<I_{b \max } \\
I_{s c \min }<I_{s c}<I_{s c \max } \\
S O C_{b \min }<S O C_{b}<S O C_{b \max } \\
S O C_{s c \min }<S O C_{s c}<S O C_{s c \max } \\
k=1,2, \ldots, N
\end{array}\right.
$$

\section{Traction Calculation}

Tram without support of traction network is a separate system. Before the design and optimization of hybrid storage system, the power and energy requirements during operation should be firstly acquired. The information can be obtained from traction calculation. The tram is subjected to traction force $\mathrm{F}$, braking force $\mathrm{B}$, and basic resistance $\omega_{0}$ and additional resistance $\omega_{j}$. The equation of additional resistance is shown as follows:

$$
\omega_{j}=\omega_{i}+\omega_{r}
$$

where $\omega_{i}$ is unit ramp resistance and $\omega_{r}$ is unit curve resistance. There are mainly three states of tram operation, traction, coasting and braking. The resultant force is calculated according to operation state, Equations (7)-(9) show the formula of traction state, coasting state, and braking state, respectively.

$$
c=\frac{F-W_{0}}{M g}-\omega_{j}
$$




$$
\begin{gathered}
c=-\frac{W_{0}}{M g}-\omega_{j} \\
c=-\frac{B+W_{0}}{M g}-\omega_{j}
\end{gathered}
$$

The equations of velocity and location are shown as follows:

$$
\left\{\begin{array}{l}
S_{t+\Delta t}=S_{t}+V_{t} \cdot \Delta t+\frac{a_{t} \cdot \Delta t^{2}}{2} \\
V_{t+\Delta t}=V_{t}+a_{t} \cdot \Delta t \\
a_{t}=\frac{c_{t} g}{1000(1+\gamma)}
\end{array}\right.
$$

The equations of power requirement are shown in Equation (11).

$$
P_{\text {req }}=\left\{\begin{array}{l}
\eta_{t} \cdot F \cdot V \\
-\eta_{r} \cdot B \cdot V
\end{array}\right.
$$

The tram traction software calculates the data based on the above formula. As Figure 5 shows, the tram conditions include marshaling, weight, motor parameters, and the line conditions include station information, station spacing, ramp conditions, curve conditions, and so on. The obtained curves of power, velocity, and energy consumption can be further used for the next step of optimization.

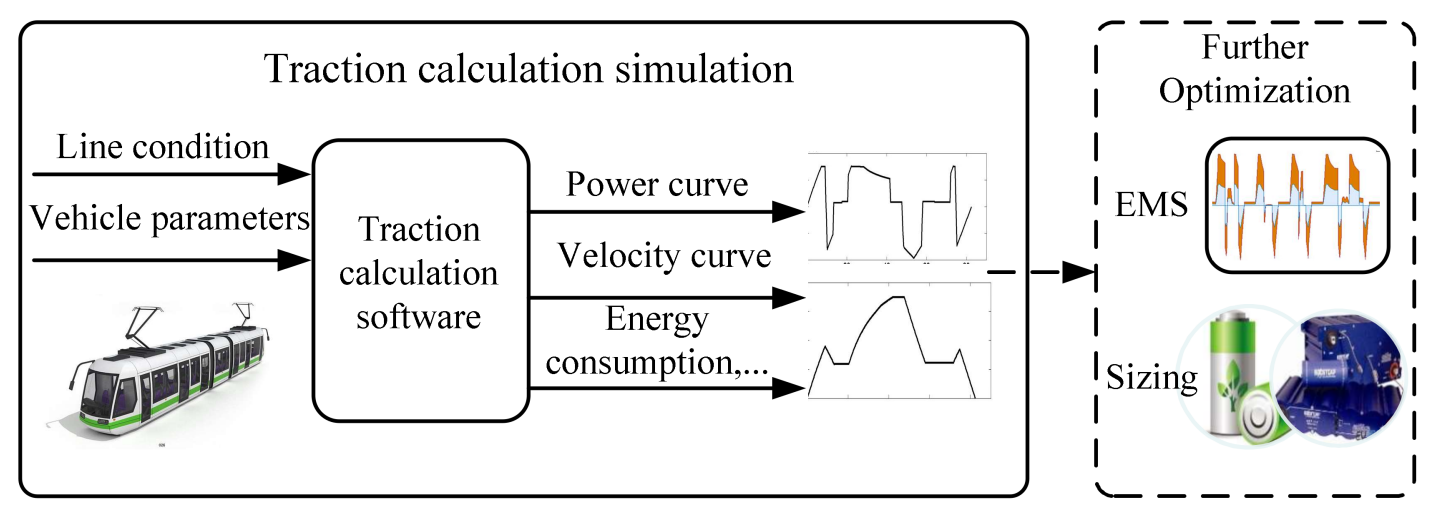

Figure 5. Constitution of tram's traction calculation software.

\section{Energy Management Strategy}

The EMS determines how the battery and supercapacitor cooperate with each other. As we know, batteries have high energy density, and supercapacitors have high power density, so to make full use of the advantages, the supercapacitor is required to provide peak traction power and absorb peak regeneration power in actual working conditions, and the battery is used to supply steady and low traction power and to absorb small part of regeneration power. In high-power applications, high discharge currents are thought to have the biggest effect on battery lifetime [19]. To avoid current impact and decrease charging time as much as possible to prolong the lifetime of the battery, its power ratio should be smaller than that of the supercapacitor.

The EMS with a fixed power ratio is the method that the ratio of output power between battery and supercapacitor is fixed. When the required power is time varying according to real working condition, each pack will be required to supply the charging or discharging power through the ratio. The power equations of traction mode and regeneration mode are shown in Equations (12) and (13), respectively.

$$
\left\{\begin{array}{l}
P_{b}=P_{r e q} \cdot p_{b} / \eta_{d c} \\
P_{S C}=P_{r e q} \cdot\left(1-p_{s c}\right) / \eta_{d c} \\
p_{b}+p_{s c}=1
\end{array}\right.
$$




$$
\left\{\begin{array}{l}
P_{b}=P_{r e q} \cdot p_{b} \cdot \eta_{d c} \\
P_{S C}=P_{r e q} \cdot\left(1-p_{s c}\right) \cdot \eta_{d c} \\
p_{b}+p_{s c}=1
\end{array}\right.
$$

where $p_{b}$ is the power ratio of battery and $p_{s c}$ is the power ratio of the supercapacitor. However, if the fixed power ratio method is put into use without improvement, in the case of the appearance of stations that consume too much energy or the charging station breaks down or any other occurrences, the supercapacitor's state of charge (SOC) will be too low that it will influence the traction mode of the tram, and the hybrid storage system cannot work normally, thus causing deceleration or the tram to shut down. To prevent such conditions from happening, the EMS should be improved where the battery provides protection for the supercapacitor in special cases. At this time, the battery's output power will increase to the highest. If the power requirement of the tram is relatively low, the battery can provide whole traction power and charge for the supercapacitor at the same time, thus going into the energy interaction mode and restraining the supercapacitor's voltage from dropping off. The power equations for the special traction mode are shown as follows:

$$
\begin{gathered}
P_{b}=P_{b \max }=I_{b \max } \cdot U_{b} \\
P_{s c}=\left\{\begin{array}{r}
\left(P_{r e q}-P_{b \max } \cdot \eta_{d c}\right) \cdot \eta_{d c} \\
, P_{r e q}>P_{b \max } \\
\left(P_{r e q}-P_{b \max } \cdot \eta_{d c}\right) / \eta_{d c} \\
, P_{r e q}<P_{b \max }
\end{array}\right.
\end{gathered}
$$

If the supercapacitor's $S O C$ is lower than the protection threshold $S O C_{s c l}$ when it is on a traction mode, the special mode will be launched, and when the $S O C$ of the supercapacitor rises to another protection threshold $S O C_{s c h}$, the special mode will be quitted. This special mode can protect the supercapacitor from the problem of insufficient power and shrink the working range, so it will help to reduce the sizing at the same time.

\section{Sizing Optimization}

\subsection{Condition Analysis}

Sizing is based on the fixed EMS shown in the last chapter. Considering the real requirements of trams and lines, there are limitations during the optimization process. The relationship of current, voltage, and power is defined as:

$$
i_{b}=\frac{U_{b}-\sqrt{U_{b}{ }^{2}-4 \cdot R_{b} \cdot P_{b}}}{2 \cdot R_{b}}
$$

where $U_{b}$ is the terminal voltage of the battery, and the limitation should be satisfied as Equation (17) shows, and similarly, the limitation caused by the supercapacitor is shown in Equation (18):

$$
\begin{gathered}
U_{b}{ }^{2}-4 \cdot R_{b} \cdot P_{b}>0 \\
U_{s c}{ }^{2}-4 \cdot R_{s c} \cdot P_{s c}>0
\end{gathered}
$$

where $U_{s c}$ is the terminal voltage of the supercapacitor. The operation state of the storage system changes frequently. To satisfy the power requirements at every moment, the real-time charging or discharging power of the battery and the supercapacitor should satisfy the power requirements and bear the loss.

$$
P_{b}+P_{s c} \geq P_{r e q}+P_{l o s}
$$


There are some charging stations along the line, which supplements the storage system. As a result, the whole energy requirement of the tram can be supplied by the initial energy that the storage system brings and the charging energy that charging stations provide, as shown in Equation (20).

$$
E_{b}+E_{S C}+E_{\text {char }} \geq E_{\text {req }}+E_{\text {los }}
$$

where $E_{b}, E_{s c}, E_{c h a r}, E_{r e q}$, and $E_{l o s}$ are the initial energy of battery and supercapacitor, the whole energy supplied in charging stations, the whole energy requirement of the tram, and the whole energy loss, respectively. If the hybrid storage system is installed on the tram, it should not be too heavy to limit the normal operation and number of passengers. The limitation is shown in the following equation:

$$
M_{b}+M_{S C} \leq M_{\lim }
$$

where $M_{b}$ and $M_{s c}$ are the weight of the battery and the supercapacitor, respectively, and $M_{\text {lim }}$ is the maximum weight limitation of the hybrid storage system. Taking cost and maintenance into consideration, the change times should not be too frequent. Therefore, the battery lifespan is required to be long enough. The criterion directly influences the ratio of the battery's output energy.

\subsection{Optimization Procedures}

Sizing optimization makes interaction effective with an EMS [20,21]. A good EMS can improve the utilization of an energy storage system and can reduce the sizing based on the system requirements. The optimization variables are the series and parallel numbers of a battery cell and a supercapacitor cell. A defined hybrid storage system and its working condition can be decided once a set of variables has been confirmed.

For a hybrid power system, many factors need to be considered in practical engineering design. First of all, due to the limited axle load of a tram, the weight of an on-board energy storage system should be limited to a certain extent to provide more passenger space. Second, a battery's lifespan is much shorter than a supercapacitor, so it may lose its function and need to be replaced before the energy storage system reaches its calendar life. To reduce the trouble caused by battery replacement and prevent replacement cost, a battery's lifespan should be considered in sizing optimization. To evaluate the battery's lifespan, the battery loss index $L I_{b}$ is introduced to indicate the loss of battery during operation. The formula is as follows:

$$
L I_{b}=\frac{365 \cdot 10 \cdot 2 \cdot C_{T} \cdot \int_{0}^{t}\left|I_{b}\right| d t}{2 \cdot L_{b} \cdot Q_{b 0}}
$$

where $C_{T}$ is the number of times that a tram operates a day. $L_{b}$ is the maximum number of cycles for the battery. The higher the $L I_{b}$, the higher the battery loss and the shorter the battery lifespan. The efficiency and weight of the energy storage system cannot be optimized at the same time. Under the same EMS, the energy consumption of the battery is certain for a whole line operation. Therefore, the less the battery configuration, the more the battery cell charges on average, and the less lifespan it has. In contrast, the larger the battery configuration, the lower the battery cell charges on average, and the longer the lifespan, but the weight of the system will increase. To optimize the weight and the battery lifespan at the same time, the multi-objective optimization is adopted and the weights of the two objectives, $\omega_{1}$ and $\omega_{2}$ are set according to the actual demands. The optimization formula of sizing is as follows:

$$
\begin{gathered}
\min F(\vec{N})=\omega_{1} \cdot M_{\text {nor }}+\omega_{2} \cdot L I_{\text {bnor }} \\
\vec{N}=N_{b s}, N_{b p}, N_{s c s}, N_{s c p}
\end{gathered}
$$


where $N_{b s}, N_{b p}, N_{s c s}$, and $N_{s c p}$ are the series and parallel numbers of the battery and the supercapacitor, respectively, and $M_{n o r}$ and $L I_{b n o r}$ are the normalized weight and battery loss index. The normalization formula is as follows:

$$
\begin{gathered}
M_{\text {nor }}(\vec{N})=\frac{M(\vec{N})-M_{\min }}{M_{\max }-M_{\min }} \\
L I_{b n o r}(\vec{N})=\frac{L I_{b}(\vec{N})-L I_{b \min }}{L I_{b \max }-L I_{b \min }}
\end{gathered}
$$

In this paper, the optimal value of the established target is obtained by optimizing the series and parallel numbers of the battery and supercapacitor, which is suitable for solving the problem by using the intelligent optimization algorithm. This paper uses an improved particle swarm optimization (PSO) algorithm. The constitutions of sizing optimization program are shown in Figure 6.

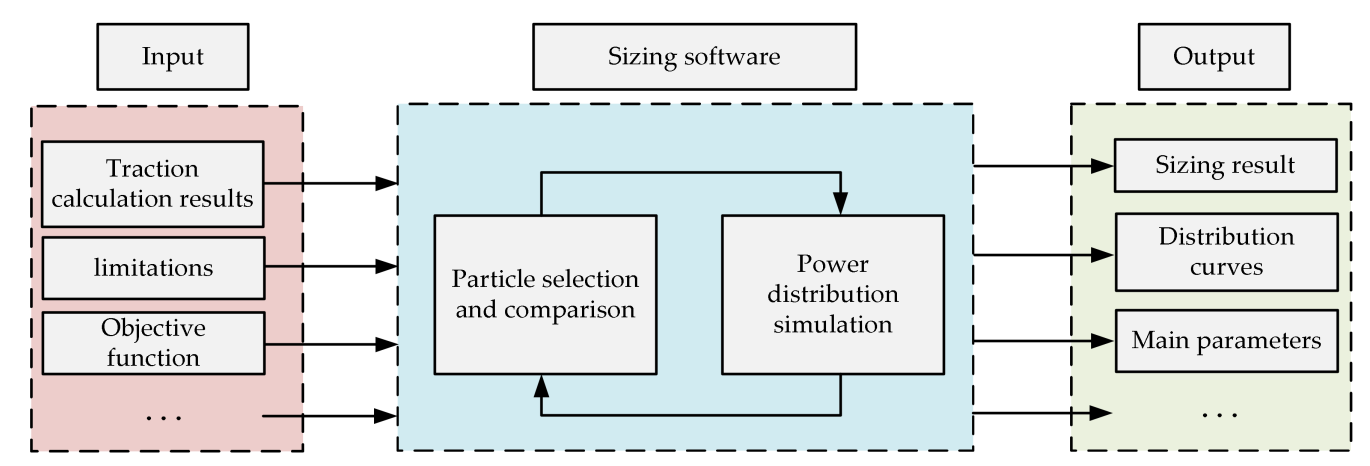

Figure 6. Constitutions of sizing optimization program.

The sizing software is divided into two parts. The main program is to select and optimize the variables in the selected value range using the optimization algorithm. Each group of variables on behalf of a certain hybrid storage system generated by the main program will enter the subprogram to perform the real-time operation simulation under the required EMS; the ones that meet the constraints are reserved and go into the next iteration, and the others are eliminated with the assignment of the minimum fitness value.

\section{Case Study}

\subsection{Tram and Line Conditions}

The paper selects a defined tram line as the study object. The total length of line is $9.44 \mathrm{~km}$, there are 11 stations, and every station has a charging device except Station 2. Data of station spacing among stop stations are shown in Figure 7.

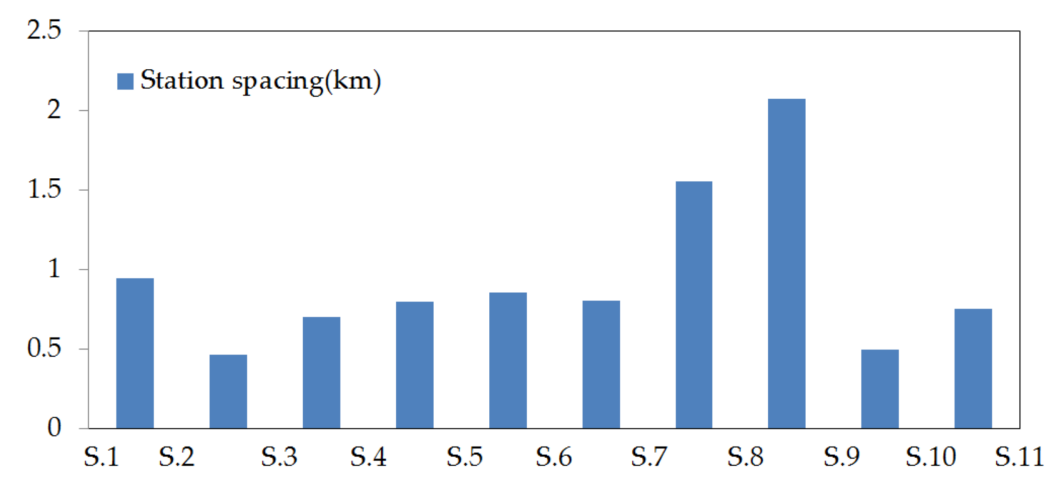

Figure 7. Histogram of station spacings of the tram line. 
It is obvious that the spacings are quite different from each other, thus making different energy consumptions among different stations. The data of speed limits and ramps should be clear. Here is an example shown in Figure 8 and the data come from the line condition between Station 7 and Station 8 .

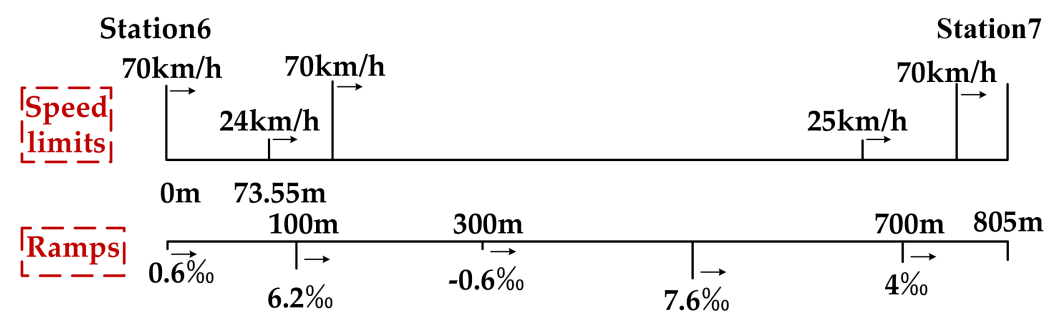

Figure 8. Data of limits and ramps between Station 7 and Station 8.

The important parameters of tram are shown in Table 1.

Table 1. Parameters of tram and line.

\begin{tabular}{cccc}
\hline Parameter & Value & Parameter & Value \\
\hline Number of motor cars & 2 & Number of motors & 4 \\
Number of trailer cars & 3 & Motor efficiency & $93.3 \%$ \\
Weight $(\mathrm{t})$ & $75(\mathrm{AW} 3)$ & Maximum velocity $(\mathrm{km} / \mathrm{h})$ & 70 \\
Bus voltage $(\mathrm{V})$ & DC750 & & \\
\hline
\end{tabular}

The parameters of tram and line are inputted into the traction calculation software, and the power demand curve is shown in Figure 9.

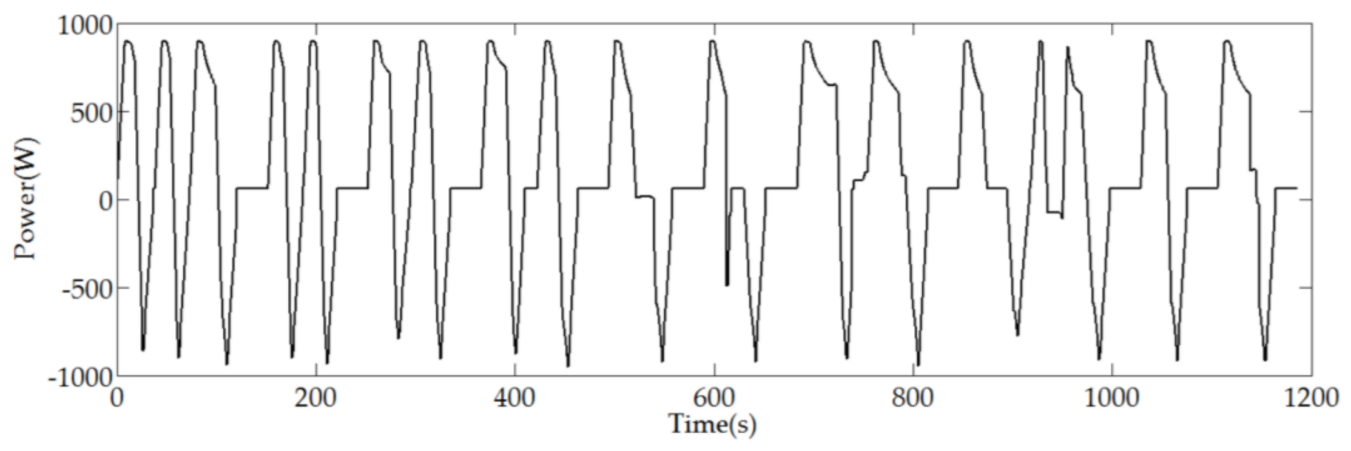

Figure 9. Power demand curve.

The maximum power is about $1 \mathrm{MW}$, and the power changes quickly. The energy consumptions with and without regeneration are shown in Figure 10.

There are great differences in energy consumption among stations. For instance, the consumption between Station 8 and Station 9 is very high. As for Station 2, it is not a charging station and the storage system cannot get supplement there, the storage system should actually store enough energy to support the operation between Station 1 and Station 3 and the energy consumption is relatively high at the same time. On the contrary, the energy consumption between Station 3 and Station 4 is very low and some other stations consume little energy as well.

Charging method also has large influence on sizing optimization. The charging process lasts $30 \mathrm{~s}$, during this time, the charging post offers 1500 A charging current to the DC bus, and the power is distributed based on the fixed power ratio, whose value is the same with operation. 


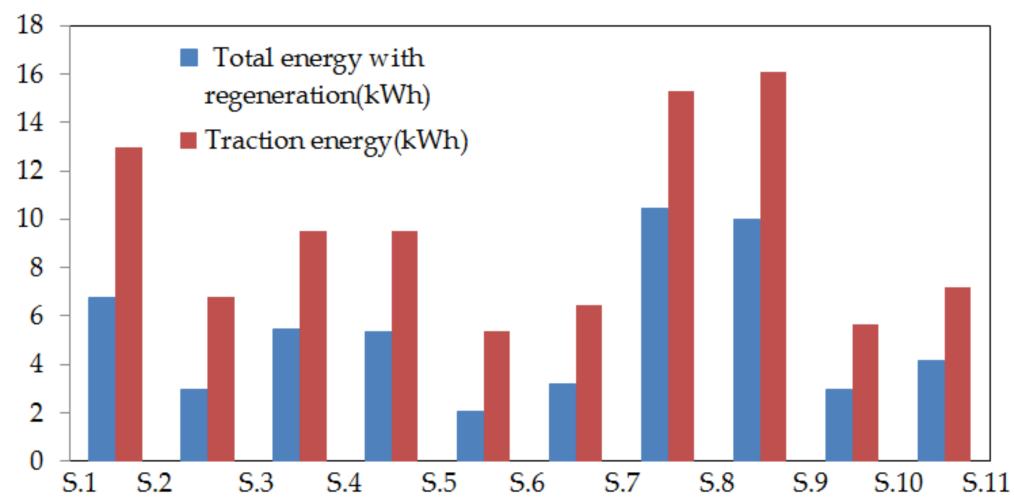

Figure 10. Energy consumption data among the stations.

\subsection{Parameter Optimization}

The parameters of EMS include power ratio and SOC thresholds and the optimization is divided into two steps. The main input parameters of the sizing optimization program under the above-mentioned condition are shown in Table 2.

Table 2. Important input parameters.

\begin{tabular}{cccc}
\hline Parameter & Value & Parameter & Value \\
\hline$S O C_{b \max }$ & $80 \%$ & $S O C_{S c \max }$ & $100 \%$ \\
$S O C_{b \min }$ & $20 \%$ & $S O C_{s c \min }$ & $25 \%$ \\
Maximum iterations & 30 & Size of particle swarm & 500 \\
$\eta_{d c}$ & $95 \%$ & & \\
\hline
\end{tabular}

The types of battery and supercapacitor have large influence on sizing result, for the energy density and power density directly decide how many cells the system allocates, the higher the energy density and power density are, the lighter the storage system is. The lithium titanate battery has the advantages of high power density and relatively long lifespan among different types of batteries, so it is popular among high power applications. After wide investigation about characteristics and price, the paper finally chooses MV06203127NTP from Microvast (Stafford, TX, USA). Maxwell (San Diego, CA, USA) has been studying and manufacturing supercapacitors for many years and the quality can be guaranteed. The paper chooses BCAP3000 from Maxwell with both long lifespan and good characteristics. The main parameters of the battery and supercapacitor are shown in Table 3 .

Table 3. Parameters of battery and supercapacitor.

\begin{tabular}{ccc}
\hline Parameter & MV06203127NTP & BCAP3000 \\
\hline Rated voltage $(\mathrm{V})$ & 2.3 & 2.7 \\
Rated capacity & $10 \mathrm{Ah}$ & $3000 \mathrm{~F}$ \\
Maximum Continuous Current (A) & $30(3 \mathrm{C})$ & 200 \\
Energy $(\mathrm{Wh})$ & 23 & 3.04 \\
Weight $(\mathrm{kg})$ & 0.3 & 0.51 \\
Cycle life & 10,000 & $2,000,000$ \\
\hline
\end{tabular}

The power ratio of the battery and supercapacitor affects the sizing of the hybrid storage system, thus affecting the charging and discharging characteristics, weight, and lifespan of the hybrid energy storage system. To verify which ratio is most suitable for the defined vehicle and the line conditions, the battery's power ratio $p_{b}$ is discretized from 0.1 to 0.9 and compared with the characteristics with the pure battery system and pure supercapacitor system, whose $p_{b}$ parameters are 1 and 0 , respectively. 
The efficiency of the energy storage system needs to be considered in the optimization. The loss of the energy storage system exists in the energy storage components, power electronic devices, and impedance components. Due to the different characteristics of the battery and supercapacitor, the EMS will impact the efficiency of energy storage system. System loss results in the overheating of the energy storage system, which will damage the lifespan of the energy storage device and cause an increase in power consumption and cost.

After the multi-objective optimization of the energy storage system with different battery power ratios, the weight, battery loss index, and the system efficiency under the optimal sizing results of each power ratio are shown in Figure 11.

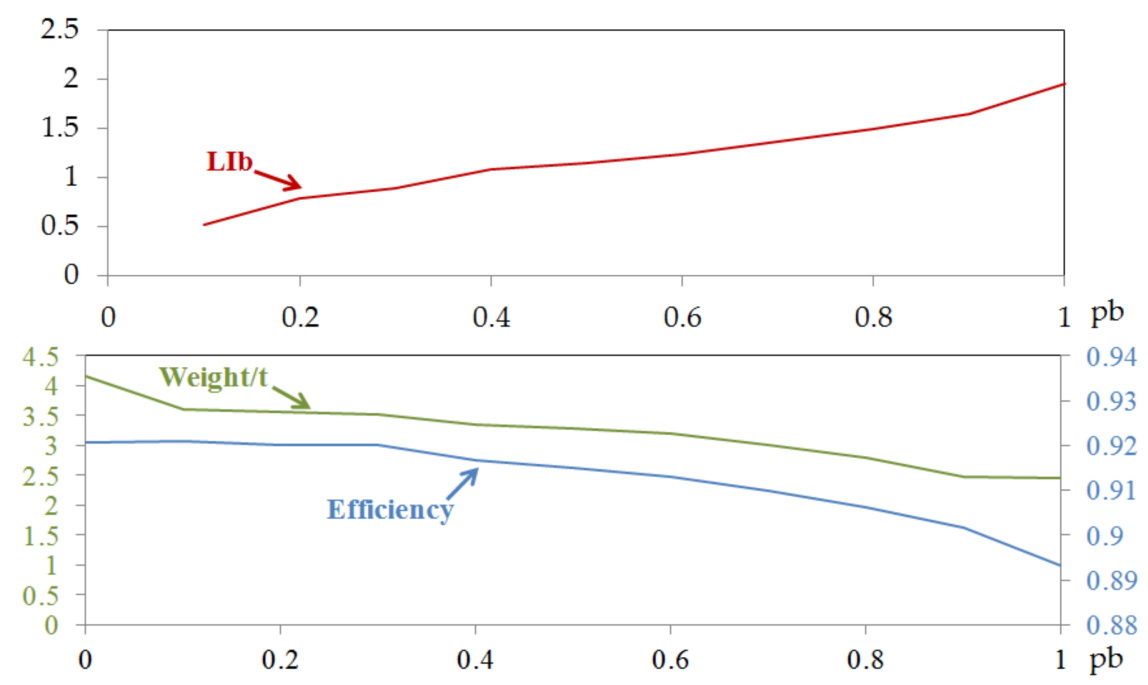

Figure 11. Weight, battery loss index, and the system efficiency curves under the optimal sizing results of each power ratio.

It can be seen from Figure 11 that the higher the power ratio of the battery, the lighter the weight and efficiency of the system. This is due to the high energy density and high internal resistance of the battery. At the same time, the battery loss index rises due to the increase of the battery output. In order to find the equilibrium among the three performances, $L I_{b}$ and weight are given the weighting of 0.7 and 0.3 , respectively. The optimal result occurs when the power ratio of battery is 0.3 .

In the next step, the parameters in the improved EMS are further optimized. The third chapter puts forward the improved management strategy with energy interaction where the thresholds of entry and exit of the special status are the supercapacitor's $S O C$ values, $S O C_{s c l}$ and $S O C_{s c h}$. The thresholds determine when the special state begins and how long it continues, and thus it determines how much the battery contributes and how long the battery's lifespan is. The parameters have direct influence on sizing optimization because they decide the working range of the storage system especially the supercapacitor. Therefore, it is necessary to optimize the two thresholds according to the demand. In this paper, all the allocations of $S O C_{s c l}$ and $S O C_{s c h}$ are traversed, and the battery loss index and the supercapacitor's maximum depth of discharge (DOD) is obtained when the $p_{b}$ is 0.3 , as shown in Figure 12a,b, respectively.

The value of $S O C_{s c l}$ is always less than that of $S O C_{s c h}$. It can be seen from Figure 12a that the smaller the $S O C_{s c l}$ and $S O C_{s c h}$, the smaller the battery loss index. Energy interaction adds an additional loss of battery, so $L I_{b}$ is higher than that without energy interaction. Conversely, the higher the $S O C_{s c l}$ and $S O C_{s c h}$, the smaller the supercapacitor's maximum DOD, and the sizing can be further optimized. However, too frequent energy interaction will lead to a lower battery lifespan, and the regeneration failure will occur if the supercapacitor's SOC is always maintained at a high level. Therefore, the loss index of the battery and the maximum DOD of the supercapacitor cannot be optimized simultaneously. 


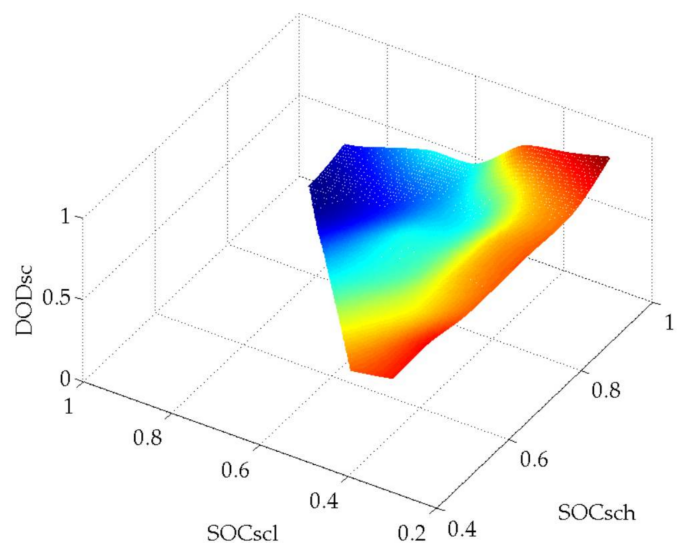

(a)

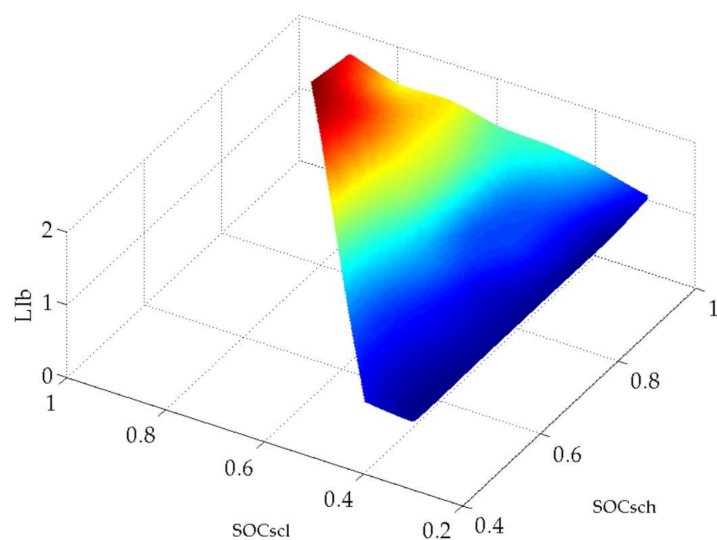

(b)

Figure 12. Three-dimensional diagrams of battery loss index and supercapacitor's maximum depth of discharge (DOD). (a) Maximum DOD of the supercapacitor. (b) Battery loss index.

The multi-objective optimization of the battery loss index and the supercapacitor's maximum DOD are both carried out with weights of 0.5 . The relationship among $S O C_{s c l}, S O C_{s c h}$, and the target value is shown in Figure 13.

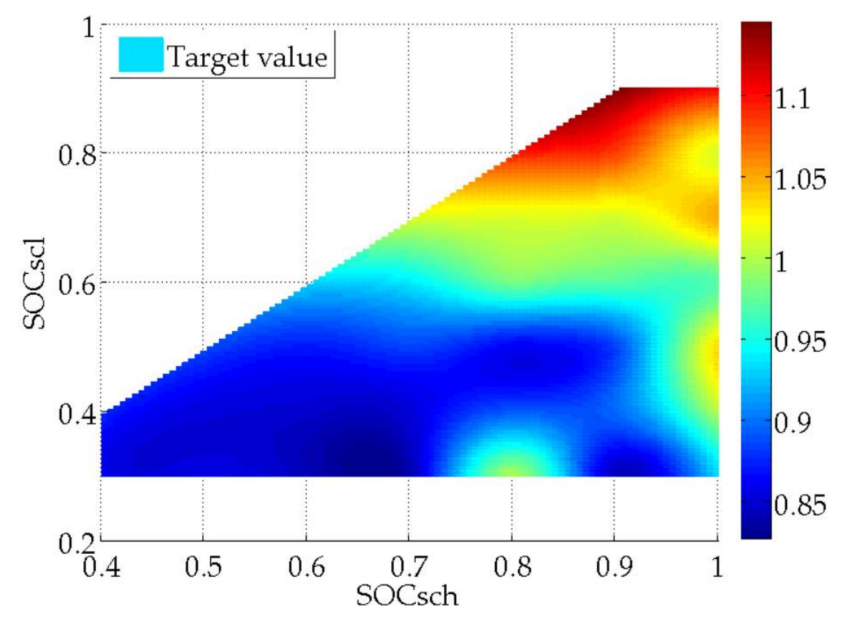

Figure 13. Three-dimensional diagram of the target value.

As is shown from Figure 13, the minimum value is concentrated in the dark blue region, and the EMS with the corresponding pair of SOC thresholds makes the best effect. After analysis and comparison, $S O C_{s c l}$ and $S O C_{s c h}$ are set to be 0.4 and 0.7 , respectively.

\subsection{Sizing Optimization}

When the power ratio of the battery is 0.3 and the SOC thresholds are 0.4 and 0.7, the supercapacitor's operation range is relatively small, so the sizing could be further optimized. Therefore, we make a sizing multi-objective optimization based on an EMS with energy interaction using the same method as the above sizing optimization, and the results of sizing under the two strategies are compared in Table 4. 
Table 4. Sizing optimization results.

\begin{tabular}{ccc}
\hline Parameter & Without Energy Interaction & With Energy Interaction \\
\hline Battery cell numbers & 4300 & 5100 \\
Supercapacitor cell numbers & 4600 & 2600 \\
Battery's energy $(\mathrm{kWh})$ & 99 & 117 \\
Supercap's energy $(\mathrm{kWh})$ & 14 & 7.9 \\
Total weight $(\mathrm{t})$ & 3.6 & 2.8 \\
$\mathrm{LI} I_{b}$ & 0.9 & 1.04 \\
\hline
\end{tabular}

As we can see from the total weight, the optimized sizing under the EMS with no energy interaction is $0.8 \mathrm{t}$ heavier than that with energy interaction, but the total energy is less mainly due to the different configuration of the supercapacitor, which has very low energy density. The energy configuration of supercapacitor with the common method is very high because it should guarantee $70 \%$ power and energy demand for the whole line including the longest spacing of the charging station in Figure 7. So, the configuration cannot be small because of the unevenly distributed charging stations. After the improvement of strategy with energy interaction, the supercapacitor can be charged by battery when the stored energy is not enough, so the pressure is lower, and the configuration result is not restrained by the longest spacing of charging station. Meanwhile, the battery's output increases, and the configuration is $20 \%$ more than that without energy interaction due to the output increase of the battery, which provides traction power and replenishes energy for the supercapacitor, and the lifespan should be kept. However, the battery's weight increases a little because of its high energy density.

To conclude, the overmuch configuration of a supercapacitor for the common method brings about the overweight of the hybrid storage system and also increases the volume and initial cost of the system. Conversely, the weight, volume, and initial cost of the optimized sizing for the strategy of energy interaction are effectively reduced. After the whole optimization of energy management and sizing, the weight of configuration has dropped by $22 \%$. This is meaningful for the onboard storage system, and the tram will have a larger space for passengers.

\subsection{Simulation Analysis}

The parameters of EMS and configuration result are described in the section above, thus the performance of hybrid storage system could be confirmed, and the simulation results of power distribution are shown in Figure 14.

The power curves and SOC curves of battery and supercapacitor are shown in Figure 14a,b, respectively. The hybrid storage system operates normally among all the stations. It works based on fixed power ratio at majority of time: the energy interaction state appeared eight times, and the state starts when the $S O C_{s c}$ is lower than 0.4 and ends when it is higher than 0.7. It is obvious that the supercapacitor can be fully charged at every charging station to guarantee the operation for the next distance. The charging time of the supercapacitor is very short for only a few seconds, while the battery charges relatively slow, so it can continue charging during the whole charging time. The battery thus can replenish energy as much as it can, so it keeps a high level of SOC during the whole operation. The superiority of the improved EMS particularly appears at the stations that consumes lots of energy. Here is a set of contrast simulation between short station spacing (between Station 5 and Station 6) and long station spacing (between Station 7 and Station 8). The power curves and SOC curves are shown in Figures 14 and 15, respectively. 


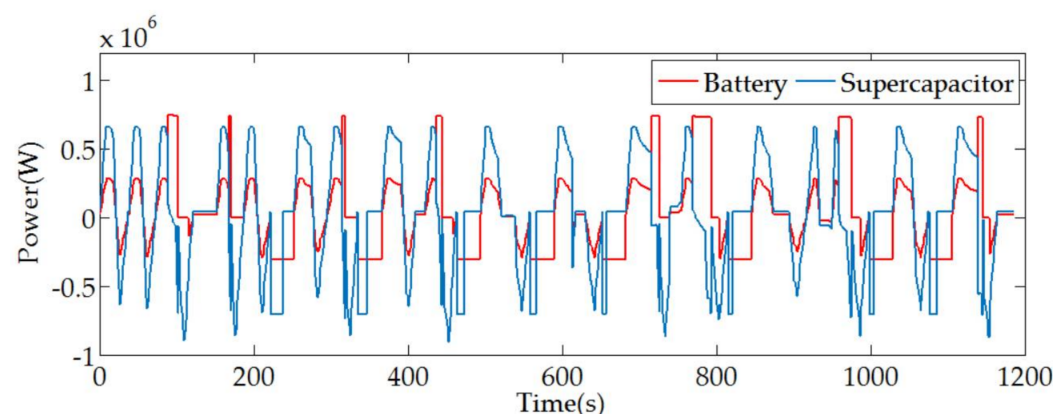

(a)

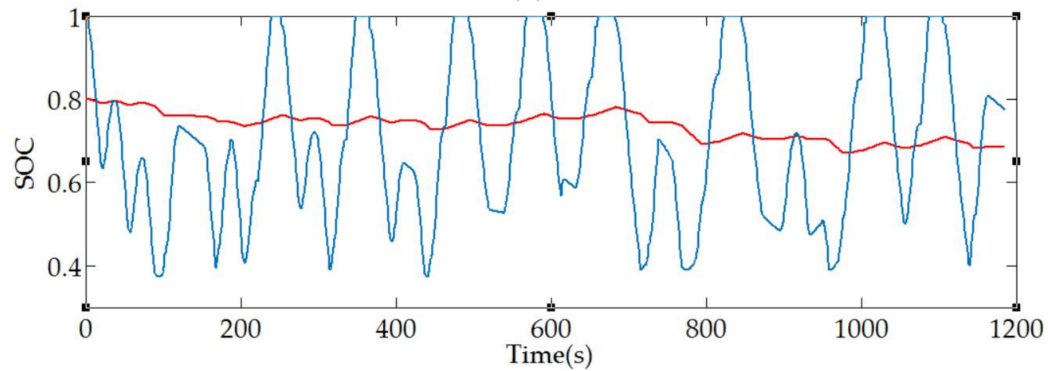

(b)

Figure 14. Simulation results. (a) Output power curves of the battery and the supercapacitor. (b) SOC curves of the battery and the supercapacitor.

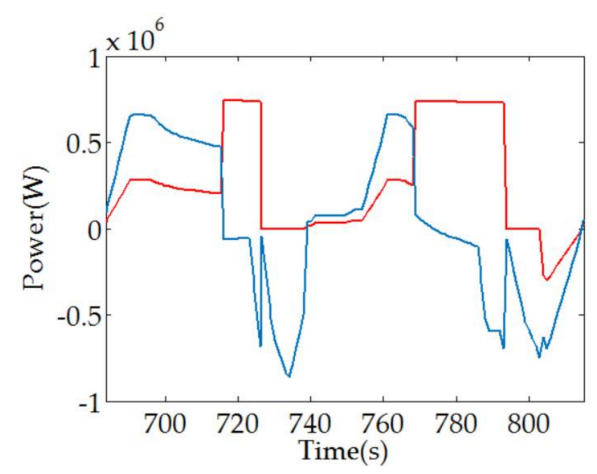

(a)

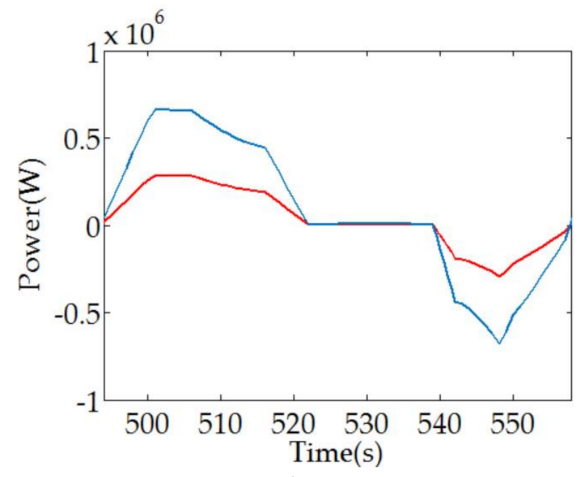

(b)

Figure 15. Output power curves of the battery and the supercapacitor. (a) Long station spacing. (b) Short station spacing.

The power distribution curves of the long spacing are shown in Figures 15a and 16a. The energy interaction state appeares two times, and it appears when the traction process is about to end. The battery can provide supercapacitor with residual power only if the reqiured traction power is less than the maximum output power of battery. The first energy interaction state and the regeneration state don't provide enough energy for the supercapacitor, so there is another energy interaction state during the next traction process. However, when the required power is higher than the battery can supply and the supercapacitor's $S O C$ is lower than $S O C_{s c l}$, the supercapacitor should output power, so the $S O C_{s c}$ can be lower than $S O C_{s c l}$. The optimized value of $S O C_{s c l}$ prevents $S O C_{S c}$ from being too low and guarantees the normal operation of the hybrid storage system. Conversely, in Figures $15 \mathrm{~b}$ and 16b, there is no energy interaction because supercapacitor consumes little energy and the SOC is higher than $S O C_{s c l}$ all the time. The supercapacitor and the battery work according to fixed power ratio. 


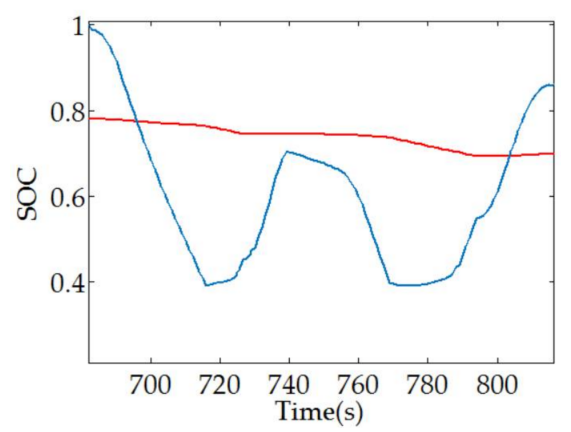

(a)

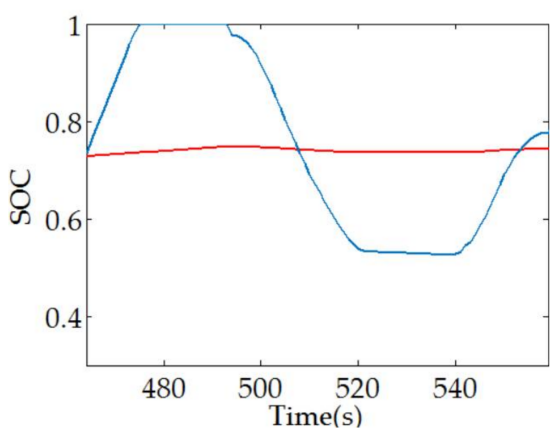

(b)

Figure 16. SOC curves of the battery and the supercapacitor. (a) Long station spacing. (b) Short station spacing.

\section{Conclusions}

In order to ensure the safe and stable operation of a tram that works without a traction network supply, the onboard hybrid storage system should be designed and optimized carefully to obtain both high energy density and high power density. In addition, as a part of the rail transit, the tram has strict requirements regarding weight and the lifespan of the hybrid storage system. In this paper, a three-step optimization has been put forward to make collaborative optimization on EMS and sizing. First, an improved EMS with energy interaction between the battery and supercapacitor has been proposed to guarantee normal operation even under emergency conditions. Second, the parameters of the EMS have been optimized considering the influence of the configuration result so that the EMS can achieve the best effect. Finally, multiple-objective sizing optimization has been made to get the optimal configuration with low weight, long lifespan, low cost, and so on. The comparation of the configuration results shows great improvement to the EMS and the weight of hybrid storage system. The performance of the optimized hybrid storage system has been verified by simulation.

Acknowledgments: This research was supported by the CRRC Qingdao Sifang Rolling Stock Research Institute.

Author Contributions: Yu Wang and Zhongping Yang conceived and designed the simulations; Yu Wang, Feng Li and Xingkun An performed the simulations and analyzed the data; Yu Wang and Fei Lin wrote the paper.

Conflicts of Interest: The authors declare no conflict of interest.

\section{References}

1. Cui, H.; Song, W.; Fang, H.; Ge, X.; Feng, X. Resonant harmonic elimination pulse width modulation-based high-frequency resonance suppression of high-speed railways. IET Power Electron. 2015, 8, 735-742. [CrossRef]

2. Simmons-Boardman Publishing Corporation. Alston Makes Advances in Traction Power. In The International Railway Journal \& Rapid Transit Review; Simmons-Boardman Publishing Corporation: New York, NY, USA, 2004.

3. Germishuizen, J.; Jockel, A.; Hoffmann, T. SyntegraTM-Next generation traction drive system, total integration of traction, bogie and braking technology. In Proceedings of the International Symposium on Power Electronics, Electrical Drives, Taormina, Italy, 23-26 May 2006.

4. De Santis, M.; Agnelli, S.; Silvestri, L.; Di Ilio, G. Characterization of the Powertrain Components for a Hybrid. Quadricycle; AIP Publishing: Melville, NY, USA, 2016.

5. Carter, R.; Cruden, A.; Hall, P. Optimizing for efficiency or battery life in a battery/supercapacitor electric vehicle. IEEE Trans. Veh. Technol. 2012, 61, 1526-1533. [CrossRef]

6. Fu, Z.; Mu, B.; Zhou, P.G. An improved logic threshold approach of energy management for a power-split hybrid electric vehicle. In Proceedings of the International Conference on Advanced Mechatronic Systems IEEE, Luoyang, China, 25-27 September 2013. 
7. Graber, G.; Galdi, V.; Calderaro, V. Sizing and energy management of on-board hybrid energy storage systems in urban rail transit. In Proceedings of the International Conference on Electrical Systems for Aircraft, Railway, Ship Propulsion and Road Vehicles \& International Transportation Electrification Conference IEEE, Toulouse, France, 2-4 November 2016.

8. Akli C, R.; Roboam, X.; Sareni, B. Energy management and sizing of a hybrid locomotive. In Proceedings of the 2007 European Conference on Power Electronics and Applications IEEE, Aalborg, Denmark, 2-5 September 2007.

9. Ferreira, A.A.; Pomilio, J.A.; Spiazzi, G. Energy Management Fuzzy Logic Supervisory for Electric Vehicle Power Supplies System. IEEE Trans. Power Electron. 2008, 23, 107-115. [CrossRef]

10. Pierre, S.; Devillers, N.; Deschinkel, K.; Péra, M.-C.; Couturier, R.; Gustin, F. Optimization of electrical energy storage system sizing for an accurate energy management in an aircraft. IEEE Trans. Veh. Technol. 2017, 66, 5572-5583.

11. Feroldi, D. Sizing and energy management for fuel cell hybrid vehicles with supercapacitors. In Proceedings of the Information Processing and Control IEEE, Cordoba, Argentina, 6-9 October 2015.

12. Bendjedia, B.; Alloui, H.; Rizoug, N. Sizing and Energy Management Strategy for hybrid FC/Battery electric vehicle. In Proceedings of the 2016 Industrial Electronics Society, Florence, Italy, 23-26 October 2016.

13. Herrera, V.I.; Milo, A.; Gaztanaga, H. Multi-Objective Optimization of Energy Management and Sizing for a Hybrid Bus with Dual Energy Storage System. In Proceedings of the 2016 IEEE Vehicle Power and Propulsion Conference, Hangzhou, China, 17-20 October 2016.

14. Shen, J.; Dusmez, S.; Khaligh, A. Optimization of Sizing and Battery Cycle Life in Battery/Ultracapacitor Hybrid Energy Storage Systems for Electric Vehicle Applications. Ind. Inf. IEEE Trans. 2014, 10, 2112-2121. [CrossRef]

15. Khaligh, A.; Li, Z. Ultracapacitor, Fuel Cell, and Hybrid Energy Storage Systems for Electric, Hybrid Electric, Fuel Cell, and Plug-In Hybrid Electric Vehicles: State of the Art. IEEE Trans. Veh. Technol. 2010, 59, 2806-2814. [CrossRef]

16. Lu, S.; Corzine K, A.; Ferdowsi, M. A New Battery/Ultracapacitor Energy Storage System Design and Its Motor Drive Integration for Hybrid Electric Vehicles. IEEE Trans. Veh. Technol. 2007, 56, 1516-1523. [CrossRef]

17. Fui, S.; Tan, C.W. A review of energy sources and energy management system in electric vehicles. Renew. Sustain. Energy Rev. 2013, 20, 82-102.

18. Johnson, V.H. Battery performance models in ADVISOR. J. Power Sources 2002, 110, 321-329. [CrossRef]

19. Lailler, P.; Zaninotto, F.; Nivet, S.; Torcheux, L.; Sarrau, J.; Vaurijoux, J.; Devilliers, D. Study of the softening of the positive active-mass in valve-regulated lead-acid batteries for electric-vehicle applications. J. Power Sources 1999, 78, 204-213. [CrossRef]

20. Zhang, X.; Mi, C.C.; Masrur, A.; Daniszewski, D. Wavelet-transform-based power management of hybrid vehicles with multiple on-board energy sources including fuel cell, battery and ultracapacitor. J. Power Sources 2008, 185, 1533-1543. [CrossRef]

21. Joong, M.; Peng, H. Power management and design optimization of fuel cell/battery hybrid vehicles. J. Power Sources 2007, 165, 819-832.

(C) 2018 by the authors. Licensee MDPI, Basel, Switzerland. This article is an open access article distributed under the terms and conditions of the Creative Commons Attribution (CC BY) license (http://creativecommons.org/licenses/by/4.0/). 